\title{
In-situ growth of iron mononitride thin films studied using x-ray absorption spectroscopy and nuclear resonant scattering
}

\author{
Mukul Gupta ${ }^{1} \cdot$ Nidhi Pandey ${ }^{1} \cdot$ Niti $^{1}$. \\ V. R. Reddy ${ }^{1}$. D. M. Phase ${ }^{1}$. Kai \\ Schlage $^{2} \cdot$ Hans-Christian Wille $^{2} \cdot$ Ajay \\ Gupta $^{3}$
}

Received: date / Accepted: date

\begin{abstract}
We studied the structural and magnetic properties of in-situ grown iron mononitride $(\mathrm{FeN})$ thin films. Initial stages of film growth were trapped utilizing synchrotron based soft x-ray absorption near edge spectroscopy (XANES) at the N $K$-edge and nuclear resonant scattering (NRS). Films were grown using dcmagnetron sputtering, separately at the experimental stations of SXAS beamline (BL01, Indus 2) and NRS beamline (P01, Petra III). It was found that the initial stages of film growth differs from the bulk of it. Ultrathin FeN films, exhibited larger energy separation between the $\mathrm{t}_{2 g}$ and $\mathrm{e}_{g}$ features and an intense $\mathrm{e}_{g}$ feature in the $\mathrm{N} K$-edge pattern. This indicates that a structural transition is taking place from the rock-salt (RS)-type FeN to zinc-blende (ZB)-type FeN when the thickness of films increases beyond $5 \mathrm{~nm}$. The behavior of such $\mathrm{N} K$-edge features correlates very well with the emergence of a magnetic component appearing in the NRS pattern at $100 \mathrm{~K}$ in ultrathin FeN films. Combining the in-situ XANES and NRS measurements, it appears that initial FeN layers grow in a RS-type structure having a magnetic ground state. Subsequently, the structure changes to ZB-type which is known to be non-magnetic. Observed results help in resolving the long standing debate about the structure and the magnetic ground state of FeN.
\end{abstract}

Keywords iron nitride - nuclear resonance scattering · N K edge spectroscopy · in-situ growth

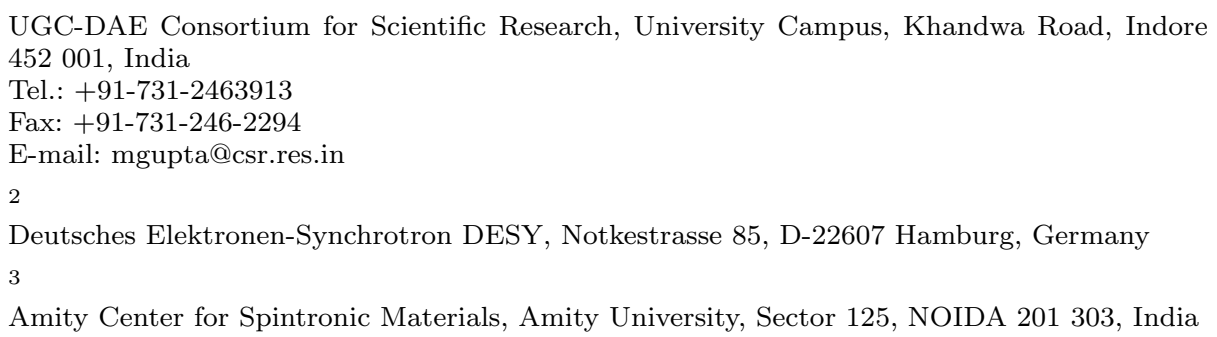




\section{Introduction}

Iron and nitrogen are among the most abundant elements on the earth and therefore iron-nitrogen compounds - $\mathrm{FeN}_{\mathrm{x}}$, are not only economical but also environment friendly. They are being investigated for applications in a variety of fields. For $\mathrm{x} \leq 0.5$ in $\mathrm{FeN}_{\mathrm{x}}$, compounds formed are magnetic and have been explored for applications as rare earth free permanent magnets, spintronic materials and also other emerging magnetic devices [1]. At $\mathrm{x}=0.12\left(\right.$ in $\left.\mathrm{FeN}_{\mathrm{x}}\right)$, the phase formed is $\alpha^{\prime \prime}-\mathrm{Fe}_{16} \mathrm{~N}_{2}$, which has been long debated to possess a giant magnetic moment, $\mathbf{M} \approx 3 \mu_{\mathrm{B}} / \mathrm{Fe}$ atom [2] and is still being a subject of intense research [3]. At $\mathbf{x}=$ 0.25 the phase formed is $\mathrm{Fe}_{4} \mathrm{~N}$, which is predicted to have almost $100 \%$ spin polarization ratio and a half-metallic character [4], its larger (than $\mathrm{Fe}$ ) $\mathbf{M} \approx 2.5 \mu_{\mathrm{B}} / \mathrm{Fe}$ atom [5-7] makes it a suitable candidate in spintronic devices and a potential alternative to Heusler alloys [8]. Between $\mathrm{x}=0.33$ to 0.5 in $\mathrm{FeN}_{\mathrm{x}}$, phases formed are $\varepsilon-\mathrm{Fe}_{3-\mathrm{y}} \mathrm{N}(0<\mathrm{y}<1)$, they are thermally stable and possess soft-magnetic properties [9] and recently exhibited for usage in Li-ion batteries [10]. At $\mathrm{x}=0.5$, the $\zeta-\mathrm{Fe}_{2} \mathrm{~N}$ phase forms [11] which has been used as a precursor to produce iron mononitride $(\mathrm{FeN})$ and iron pernitride $\left(\mathrm{FeN}_{2}\right)$ by application of high-pressure and high-temperature (HPHT) [12].

$\mathrm{N}$ rich phases of iron-nitrogen compounds $\left(\mathrm{x}>1\right.$ in $\left.\mathrm{FeN}_{\mathrm{x}}\right)$ were theoretically predicted for a while, e.g. the spinel nitride $\mathrm{Fe}_{3} \mathrm{~N}_{4}[13,14]$ and the pernitride $\mathrm{FeN}_{2}$ [15], only very recently the $\mathrm{FeN}_{2}$ phase has been synthesized experimentally under HPHT $(\mathrm{T} \approx 2000 \mathrm{~K}, \mathrm{P} \approx 50 \mathrm{GPa})[12,16]$ and by raising the pressure above $100 \mathrm{GPa}$, the $\mathrm{FeN}_{4}$ phase was also evidenced by Bykov et al. [16]. Very recently even higher $\mathrm{N}$ phases like $\mathrm{FeN}_{6}$ and $\mathrm{FeN}_{8}$ have been predicted [17]. Polymeric nitrogen chains present in $\mathrm{N}$ rich $\mathrm{FeN}_{\mathrm{x}}$ compounds makes them very attractive as high energy density materials (HEDMs), however, their synthesis under ambient temperature and pressure remains a challenge.

While magnetic $\mathrm{FeN}_{\mathrm{x}}$ compounds already started to get attention in early 1950s [18], experimental works on the mononitride phase can only be traced back in the last three decades or so [19-22]. FeN compounds were extensively studied by Schaff et al. [23-26] in late 1990s. Subsequently, FeN thin films were synthesized using ion beam sputtering [27], dc/rf magnetron sputtering [28-38], pulsed laser deposition (PLD) [39-42], high power impulse magnetron sputtering [43], nitrogen plasma assisted molecular beam epitaxy (MBE) [44-48] and very recently under HPHT $[12,16,49-51]$. From applications points of view, the mononitride FeN is also very interesting as its oxidation resistance makes it a effective catalyst in chemical reactions $[52,53]$, it can be used as a precursor to yield magnetic phases in a controlled way $[38,44,54,55]$ and also in biomedical applications [17]. However, the fundamental understanding of mononitride FeN compound is still not well understood and variances can be seen between theory and experiments in terms of the lattice parameter (LP), structure and the magnetic ground state.

Considering recent theoretical works $[17,56-61]$ and comparing with experimental works, various possibilities emerge about the structure and the magnetic ground state of FeN (i) $\gamma^{\prime \prime}$-FeN with zinc-blende $(\mathrm{ZB})$-type structure $(\mathrm{LP} \approx 4.3 \AA$ ) and a nonmagnetic (NM) ground state (ii) $\gamma^{\prime \prime \prime}$-FeN with rock-salt (RS)-type structure $(\mathrm{LP} \approx 4.5 \AA)$ and a ferromagnetic $(\mathrm{FM})$ or antiferromagnetic (AFM) ground state (iii) NiAs-type structure with a FM ground state (iv) CsCl-type structure with NM ground state (iv) wurtzite-type structure with a NM ground state (v) 
MnP-type structure at very high pressure. Among these, $\mathrm{FeN}$ in $\mathrm{CsCl}$, wurtzite and $\mathrm{MnP}$ structures has not yet been synthesized experimentally, NiAs-type structure was experientially evidenced recently under HPHT [12,16, 49-51]. Under ambient temperature and pressure conditions only the ZB and RS-type FeN have been evidenced hitherto, however the existence of RS-type FeN is still debated experimentally. Recent first-principles calculations carried out by $\mathrm{Li}$ et al. [56] predict that the total energy for ZB-FeN (non spin polarized) at $-16.893 \mathrm{eV}$ is only slightly lower than for RS-FeN (spin polarized) at $-16.892 \mathrm{eV}$. This energy is significantly higher for all other FeN phases mentioned above. Therefore, at $\mathrm{T}=0 \mathrm{~K}$ and $\mathrm{P}=$ $0 \mathrm{GPa}$, the preferred phase is ZB-type FeN followed by RS-type FeN.

From a review of recent experimental works probing the magnetic ground state of FeN, it can be seen that irrespective of its LP ( 4.3 or $4.5 \AA$ ), FeN remains nonmagnetic down to $2 \mathrm{~K}$. An exception to this, are studies performed by Usui and Yamada [41,42] and earlier works of Nakagawa [20], Suzuki [21] and Hinomura [22] et al. In all these works a magnetic phase was found at low temperature but along with an oxide phase. Usui and Yamada [41,42] deposited FeN films using PLD and by varying the nitrogen partial pressure they synthesized ZB-type $\gamma^{\prime \prime}$-FeN and RStype $\gamma^{\prime \prime \prime}$-FeN. In these works low temperature (down to $5 \mathrm{~K}$ ) ${ }^{57} \mathrm{Fe}$ Mössbauer spectroscopy measurements were carried out and it was concluded that $\gamma^{\prime \prime}$-FeN remain $\mathrm{NM}$ and $\gamma^{\prime \prime \prime}$-FeN become AFM at low temperature. The Néel temperature $\left(\mathrm{T}_{\mathrm{N}}\right)$ estimated for $\gamma^{\prime \prime \prime}$-FeN films was $220 \mathrm{~K}$. In addition to the AFM component with a hyperfine field $\left(\mathbf{B}_{\mathrm{hf}}\right)$ of about $30 \mathrm{~T}$ an additional component with $\mathbf{B}_{\mathrm{hf}}=49 \mathrm{~T}$ was also observed. Generally, such component stems from an oxide phase $[20,22]$ therefore, the purity of these samples was questionable.

Jouanny et al. [32] prepared FeN thin films having LP $\approx 4.5 \AA$ using sputtering at $373 \mathrm{~K}$ and from their zero-field-cooled and field-cooled (1 T) magnetization measurements they could not find the presence of any magnetic order. J. Pak et al. [47] studied the growth behavior of epitaxial FeN thin films deposited using $\mathrm{N}$ plasma assisted MBE and from their in-situ reflection high energy electron diffraction (RHEED) measurements they found that the LP of ultrathin FeN films was about $4.52 \AA$. Up to about 53 monolayers (ML), the LP of FeN films remained at this value but for a $140 \mathrm{ML}$ thick $(\approx 60 \mathrm{~nm})$ FeN film, the LP reduced to $4.32 \AA$. This reduction in LP was explained in terms of an intrinsic in-plane tensile stress that generates during the growth of the film. They also did magnetization and susceptibility measurements on the $60 \mathrm{~nm}$ thick sample down to $5 \mathrm{~K}$ and could not find the presence of any magnetic order. Gupta et al. [34] prepared FeN films with $\mathrm{LP} \approx 4.5 \AA$ using sputtering at $300 \mathrm{~K}$ and did ${ }^{57} \mathrm{Fe}$ Mössbauer spectroscopy measurements down to $5 \mathrm{~K}$ and could not find any magnetic ordering even after applying a magnetic field of $5 \mathrm{~T}$. It may be noted that the LP of FeN films seems to vary with the growth temperature $\left(\mathrm{T}_{\mathrm{S}}\right)$, when $\mathrm{T}_{\mathrm{s}}<400 \mathrm{~K}, \mathrm{LP} \approx 4.5 \AA$ and between $400-500 \mathrm{~K}$ its was $\mathrm{LP} \approx 4.3 \AA$.

Above experimental results indicate that ultrathin FeN films may behave differently than their thicker counterparts. When the growth of FeN films starts their LP is large and an intrinsic in-plane tensile stress forces it to reduce. In such a situation, it can be assumed that initial FeN layer grow with a RS-type motif which changes to ZB-type when film thickness increases beyond a critical value. This critical thickness can be changed by varying the deposition conditions. However, to prove this point, low temperature magnetic measurements need to be done on ultrathin films, preferably during in-situ growth to avoid surface contamination. 


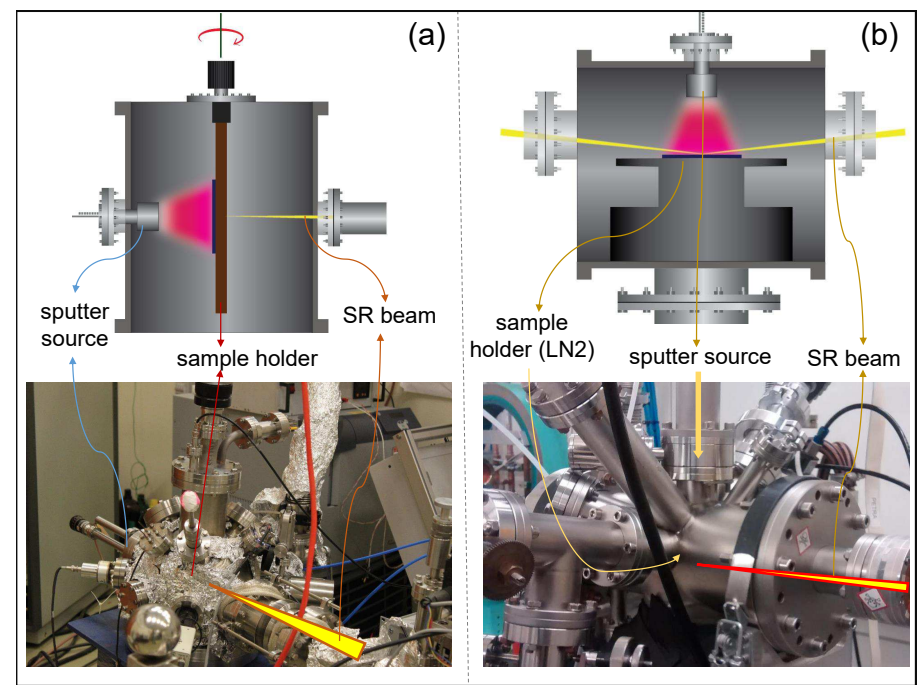

Fig. 1 A schematic diagram and a photograph of the in-situ system prepared at the soft x-ray absorption spectroscopy beamline BL01 at Indus 2 (a) and at the nuclear resonance scattering beamline P01 at Petra III (b). In both cases a $\phi 1$ inch magnetron source was used.

Hitherto, low temperature magnetization measurements were performed only on thick FeN films (> $50 \mathrm{~nm})$.

Magnetization and structural measurements at low temperatures on ultrathin films combined with in-situ thin film growth is a challenging task. In the present work we achieved this by preparing in-situ systems capable of doing x-ray absorption near edge spectroscopy (XANES) measurements at $\mathrm{N} K$-edge and nuclear resonant scattering (NRS) measurements. It is known that $\mathrm{N} K$-edge XANES can provide reliable information about the coordination between metal and $\mathrm{N}$ atoms [62] and NRS is a technique which is analogues to Mössbauer spectroscopy thereby providing the information about the local magnetic structure [63]. In both cases FeN films were grown using a similar magnetron sputtering source ( $\phi 1$ inch) and under similar deposition conditions. From N $K$-edge XANES measurements, we observed a structural transition taking place between 5-10 nm and low temperature $(100 \mathrm{~K})$ NRS measurements also exhibited the presence of a magnetic component at similar thicknesses. These first ever in-situ N $K$-edge and NRS measurements clearly reveal that ultrathin FeN films grow in the RS-type structure and are magnetic at $100 \mathrm{~K}$. When the thickness of FeN film increases beyond few nm, the structure changes to ZB-type FeN which has a non-magnetic ground state.

\section{Experimental}

In order to study the early stages of film growth, a magnetron sputtering system was mounted separately at the soft x-ray absorption spectroscopy (SXAS) beamline BL01 [64] of Indus 2 synchrotron radiation (SR) source at RRCAT, Indore, India and at the NRS beamline P01 [65] of Petra III SR radiation source at 
DESY, Hamburg, Germany. In both cases a $\phi 1$ inch magnetron source (AJA Int. Inc.) with $\mathrm{Fe}$ or ${ }^{57} \mathrm{Fe}$ target was used and keeping experimental conditions similar, FeN films of thickness ranging from sub nm to few tens of nm were deposited. Nitrogen alone was used to sputter the iron target. By using nitrogen alone as the sputtering medium (instead of a mixture of $\mathrm{Ar}+\mathrm{N}_{2}$ ), single phase $\mathrm{FeN}$ films can be synthesized in reactive dc magnetron sputtering(dcMS) [20,34,35,38]. After growing a film of a particular thickness, XANES or NRS measurements were performed. Prior to in-situ experiments, few test samples were deposited (on Si and $\mathrm{SiO}_{2}$ substrates) to determine the deposition rates (using x-ray reflectivity), the crystalline structure using x-ray diffraction (XRD, Bruker D8 Advance using $\mathrm{Cu}-K_{\alpha}$ x-rays) and the magnetic structure using room temperature conversion electron Mössbauer spectroscopy (CEMS). The isomer shifts (IS) were measured with respect to $\alpha$-iron. The details of the $i n-s i t u$ experimental stations exclusively prepared in this work are described below.

\subsection{In-situ system at SXAS beamline}

In the ultra-high vacuum (UHV) experimental station of SXAS beamline, a magnetron sputtering source was installed. This source was mounted as shown in the schematic diagram in fig. 1 (a). A photograph of the experimental chamber with the sputter source is also shown in fig. 1 (a). Using this source FeN films were deposited on a Si substrate facing the source and then the substrate was rotated along its axis so as to allow the SR x-rays to fall directly on to the deposited sample. A mass flow controller was also mounted to control the gas flow precisely. Using $\mathrm{N}_{2}$ (purity 99.9995\%) alone as the sputtering medium, an iron target (purity $99.995 \%$ ) - $\phi 1$ inch and thickness $0.5 \mathrm{~mm}$ was sputtered. The base pressure in the vacuum chamber was about $2 \times 10^{-8}$ mbar and with $\mathrm{N}_{2}$ gas flowing at about $20 \mathrm{sccm}$, the pressure during deposition was about $5 \times 10^{-3}$ mbar.

The sputter source was equipped with a shutter so that the actual film deposition can be started after stabilizing the source conditions. Prior to in-situ experiments, Fe target was pre-sputtered to remove surface contaminations. The sputtering power used was about $15 \mathrm{~W}(450 \mathrm{~V} \mathrm{dc})$ and the target - substrate distance was about $5 \mathrm{~cm}$. All samples were deposited at ambient temperature without any intentional heating on a clean Si substrate. After each deposition the chamber was evacuated back to UHV conditions and XANES measurements at the $\mathrm{N} K$ edges were carried out in the total electron yield mode. Typically, a N $K$-edge scan takes about a couple of minutes and a number of scans were averaged to improve the data reliability and statistics. The energy resolution at the $\mathrm{N} K$-edge is better than $0.2 \mathrm{eV}$. The effectiveness of such in-situ growth using SXAS has been amply demonstrated during the growth of $\mathrm{Cr}$ [66], $\mathrm{TiO}_{2}$ [67], $\mathrm{TiN}$ [68] and very recently for Fe films on $\mathrm{MgO}$ [69].

\subsection{In-situ system at NRS beamline}

Similarly, for in-situ NRS measurements also a MS source with target diameter of 1 inch was installed in a vacuum chamber as shown in the schematic diagram and photograph in fig.1 (b). Here instead of natural Fe, a ${ }^{57} \mathrm{Fe}$ target with about $95 \%$ 
abundance and $99.95 \%$ purity was used. The thickness of this target was $0.4 \mathrm{~mm}$. The sample holder was either held at ambient temperature $(300 \mathrm{~K})$ or cooled with liquid nitrogen $\left(\mathrm{LN}_{2}\right)$ flowing continuously in the copper reservoir holder. The temperature achieved at the substrate surface was about $100 \mathrm{~K}$. The base pressure achieved in the chamber was about $3 \times 10^{-8}$ mbar (after a bake out). All other deposition conditions were kept similar as described in section 2.1. Samples were deposited on a sapphire substrate.

Here also after depositing a film, the chamber was evacuated again to UHV conditions and then NRS and x-ray (electronic) reflectivity (XRR) was measured. Since both NRS and XRR measurements can be done, almost simultaneously (one after the other within few minutes), the information about the magnetic structure and sample thickness, density, roughness etc. can be obtained in a unique way. The SR beam was tuned to ${ }^{57} \mathrm{Fe}$ nuclear resonance energy of $14.4 \mathrm{keV}$. Utilizing the pulse structure of SR beam, the prompt scattering was used to record the XRR pattern, in the $\theta-2 \theta$ mode and NRS was recorded using a multi-channel analyzer between 10 to $150 \mathrm{~ns}$ in the grazing incidence geometry. The angle of incidence during NRS measurements was kept at the critical angle $\approx 0.156^{\circ}$. The critical angle was determined precisely by measuring the nuclear resonance reflectivity (not shown). The details of the beamline can be in found in $[65,70]$ and about the NRS of SR in [63,71-74].

\section{Results and Discussion}

\subsection{Structural and magnetic characterization of FeN thin film}

Prior to in-situ experiments, preliminary characterization of a $50 \mathrm{~nm}$ thick FeN sample (deposited simultaneously on a native $\mathrm{Si}$ and $\mathrm{SiO}_{2}$ substrates) was carried out to understand its structural and magnetic properties. Samples deposited Si or $\mathrm{SiO}_{2}$ substrates yielded similar results. Shown in fig.2 (a) is the XRD pattern of this sample (on $\mathrm{SiO}_{2}$ substrate) exhibiting a broad peak around $2 \theta=34.9^{\circ}$ with a grain size of about $12.5( \pm 0.5) \mathrm{nm}$. This peak can be assigned to $\mathrm{FeN}(111)$ and the LP comes out to be $4.45 \AA$. Generally, the LP of FeN films has been found anywhere between 4.3 to $4.55 \AA$ and when FeN films are deposited at low $\mathrm{T}_{\mathrm{s}}(<$ $400 \mathrm{~K}), \mathrm{LP} \approx 4.5 \AA[32,34,75]$ and at high $\mathrm{T}_{\mathrm{s}}, \mathrm{LP}$ reduces to about $4.3 \AA[47$, 75]. Therefore, from our XRD measurements, we can confirm the formation of mononitride FeN phase.

The $\mathrm{N} K$-edge XANES pattern of this test sample (on $\mathrm{SiO}_{2}$ substrate) is shown in the inset fig.2 (a). Here, prominent features are labelled as $a, b$ and $c$; $a$ and $b$ correspond to transition from $\mathrm{N} 1 s$ to hybridized states of Fe $3 d$ and $\mathrm{N}$ $2 p$. Features $a$ and $b$ can be assigned as $\mathrm{t}_{2 g}$ and $\mathrm{e}_{g}$ in the octahedral and e and $\mathrm{t}_{2}$ in the tetrahedral symmetry as explained later in section 3.3. Higher energy feature $c$ is due to electronic transition from N1s to a hybridized N2p and Fe $4 s p$ states [62]. Although N $K$-edge XANES has been used as a finger print to identify the electronic structure of early transition metal nitrides, such measurements in FeN system are almost nonexistent. In our recent work, we found that the intensity of feature $b$ becomes almost negligible (compared to feature $a$ ) [35] in 150-200 nm thick FeN films deposited at $300 \mathrm{~K}$. Generally, in octahedral symmetry both $a$ and $b$ features are clearly resolved e.g. in case of TiN [76] and $\mathrm{CrN}[77,78]$. In fact $\mathrm{N} K$ - 


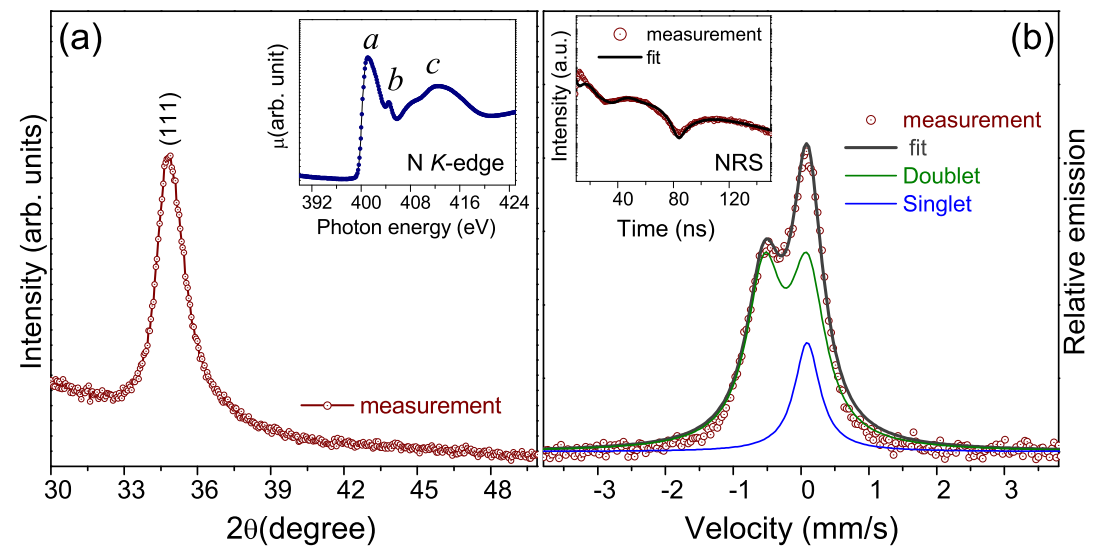

Fig. 2 X-ray diffraction pattern of a $50 \mathrm{~nm}$ FeN film grown at $300 \mathrm{~K}$ on an amorphous $\mathrm{SiO}_{2}$ substrate (a) and the inset shows the N K-edge XANES pattern of this sample. Conversion electron Mössbauer spectrum of a $50 \mathrm{~nm}{ }^{57} \mathrm{FeN}$ thin film grown at $300 \mathrm{~K}$ on an amorphous $\mathrm{SiO}_{2}$ substrate (b) and the NRS pattern of a similar sample is shown in the inset of (b).

Table 1 Fitting parameters obtained from fitting of CEMS measurements for a ${ }^{57} \mathrm{FeN}(50 \mathrm{~nm})$ thin film sample and compared with recent reported values. Here, isomer shift (IS) were measured with respect to $\alpha$-Fe and $\triangle \mathrm{IS}$ is the difference between IS of singlet (S) and doublet (D), QS denotes to quadrupole splitting, and R.A. is relative area of S or D.

\begin{tabular}{cccccccc}
\hline \hline $\begin{array}{c}\text { Thickness } \\
(\mathrm{nm})\end{array}$ & $\begin{array}{c}\text { R.A.(S) } \\
(\%)\end{array}$ & $\begin{array}{c}\text { R.A.(D) } \\
(\%)\end{array}$ & $\begin{array}{c}\text { IS(S) } \\
(\mathrm{mm} / \mathrm{s})\end{array}$ & $\begin{array}{c}\text { IS(D) } \\
(\mathrm{mm} / \mathrm{s})\end{array}$ & $\begin{array}{c}\text { QS(D) } \\
(\mathrm{mm} / \mathrm{s})\end{array}$ & $\begin{array}{c}\triangle \text { IS } \\
(\mathrm{mm} / \mathrm{s})\end{array}$ & Ref. \\
\hline 36 & 12.4 & 87.6 & -0.02 & 0.22 & 0.47 & 0.24 & {$[45,46]$} \\
18 & 24 & 76 & 0.006 & 0.26 & 0.46 & 0.27 & {$[79]$} \\
$50 \pm 0.5$ & $20 \pm 1$ & $80 \pm 1$ & $0.09 \pm 0.002$ & $-0.22 \pm 0.01$ & $0.64 \pm 0.01$ & 0.31 & this work \\
\hline \hline
\end{tabular}

edge features have been compared in fcc $\mathrm{CrN}$ and hcp $\mathrm{Cr}_{2} \mathrm{~N}$ and it was anticipated that due to octahedral crystal field, $\mathrm{N} K$-edge features show splitting in CrN but in $\mathrm{Cr}_{2} \mathrm{~N}$, no such splinting can be seen in absence of octahedral crystal field [77]. Therefore, N $K$-edge XANES can be used to distinguish between ZB and RS-type FeN, as demonstrated from our in-situ measurements presented in section 3.3.

The magnetic state of another test sample $\left(50 \mathrm{~nm}\right.$ thick ${ }^{57} \mathrm{FeN}$ film on $\mathrm{SiO}_{2}$ substrate) was measured using CEMS as shown in fig. 2 (b). It shows an asymmetric peak typically found in FeN films and the absence of sextet confirms $\mathbf{B}_{\mathrm{hf}}=0 \mathrm{~T}$. This peak has been fitted using a number of combinations of singlet (S) or doublet (D) such as: (i) two $\mathrm{S}[20,32,34,45]$ (ii) one $\mathrm{S}$ and one $\mathrm{D}[45,46,79]$ (iii) two $\mathrm{S}$ and one D [25]. In all these studies the component $\mathrm{S}$ with isomer shift (IS) $\approx 0 \mathrm{~mm} / \mathrm{s}$ has always been assigned to Fe coordinated to four N neighbors as in ZB-type FeN. But the assignment of the second component (either $\mathrm{S}$ or D) remains a matter of debate and still its origin and nature is not clear. In earlier works, this component was ascribed as RS-type FeN but in recent works the existence of RS-type FeN 
was ruled out from low temperature bulk magnetization measurements [32] and also from low temperature CEMS measurements in thick ( $>50 \mathrm{~nm}$ ) FeN films [34].

As of now, the emergence of second component (S or D) in CEMS spectrum of $\mathrm{FeN}$ has been explained in terms of vacancies or defects in ZB-type FeN. Andrzejewska et al. [46] attempted to address these issues in detail and concluded that CEMS spectrum can be best fitted using a S+D combination [46]. Therefore adopting a similar approach, we fitted our CEMS spectrum assuming one S and one D combination as shown in fig. 2 (b) and obtained fitting parameters are given in table 1. In this table, our CEMS fitting parameters are compared with those reported in literature for MBE grown ${ }^{57} \mathrm{FeN}$ films $[45,46,79]$. It can be seen that our parameters follow a similar trend as (i) the IS of $\mathrm{S}$ is about $0 \mathrm{~mm} / \mathrm{s}$ (ii) the IS of D is higher than that of $\mathrm{S}$ (iii) the relative area (R.A.) of D is significantly higher than S. However, some small difference can be seen in terms of slightly higher values IS of S and D and also QS of D in our case. These difference may arise as our samples were grown using reactive sputtering at $300 \mathrm{~K}$ while MBE grown samples in reference $[45,46]$ were grown at $323 \mathrm{~K}$ and that in reference [79] at $423 \mathrm{~K}$.

Contrary to the CEMS, the NRS measurements (on a third generation synchrotron radiation source) can be carried out within few minutes even when the thickness of sample is only few nm. However, unlike the CEMS, the NRS pattern lacks distinguished features and therefore difficult to follow. Therefore, a typical NRS pattern of another FeN sample is compared for illustration purpose in the inset of fig. 2 (b). The featureless pattern confirms that this sample is non-magnetic. Details of NRS results are discussed in section 3.4. Therefore, our XRD, XANES, and CEMS measurements confirm that the $50 \mathrm{~nm}$ FeN sample has a ZB-type structure with a LP of $4.45 \AA$ and it is non-magnetic (as $\mathbf{B}_{\mathrm{hf}}=0 \mathrm{~T}$ ) at $300 \mathrm{~K}$. This result is in agreement with recent works on FeN thin films $[32,45-47,79]$.

\subsection{In-situ NRS measurements at $300 \mathrm{~K}$}

Figure $3(\mathrm{a}, \mathrm{b})$ shows the XRR and NRS patterns taken during in-situ deposition of ${ }^{57} \mathrm{FeN}$ thin film for various film thicknesses. As films become thick, the frequency of oscillation increases in the XRR pattern signifying that the thickness of films is increasing. The fitting of XRR pattern was carried out using Parratt32 software [80] and it reveals that film roughness is about 1-2 $\mathrm{nm}$ and the estimated film density matches well with FeN films. As expected, film thickness increased linearly with deposition time. The deposition rate was about $0.055 \mathrm{~nm} / \mathrm{s}$.

The NRS spectra of in-situ grown FeN films are featureless except for a broad oscillation that shifts to lower times as thickness increases. Such broad oscillation arise due to dynamical beats and hyperfine parameters (QS and IS), as explained in section 3.4. The absence of beating patterns arising due to $\mathbf{B}_{\mathrm{hf}}$ signifies that FeN films are non-magnetic, irrespective of the film thickness. The fitting of NRS pattern was carried out using REFTIM software [81]. In order to fit NRS spectra, we adopted a similar model that was applied to fit the CEMS spectrum using a combination of one S and one D. Fitted parameters are shown in table 2. Here, it may be noted that unlike the CEMS, the determination of absolute values of IS is not possible in the NRS as it requires adding a single-line reference sample to the experimental setup which is spatially separated from the absorber by a distance 


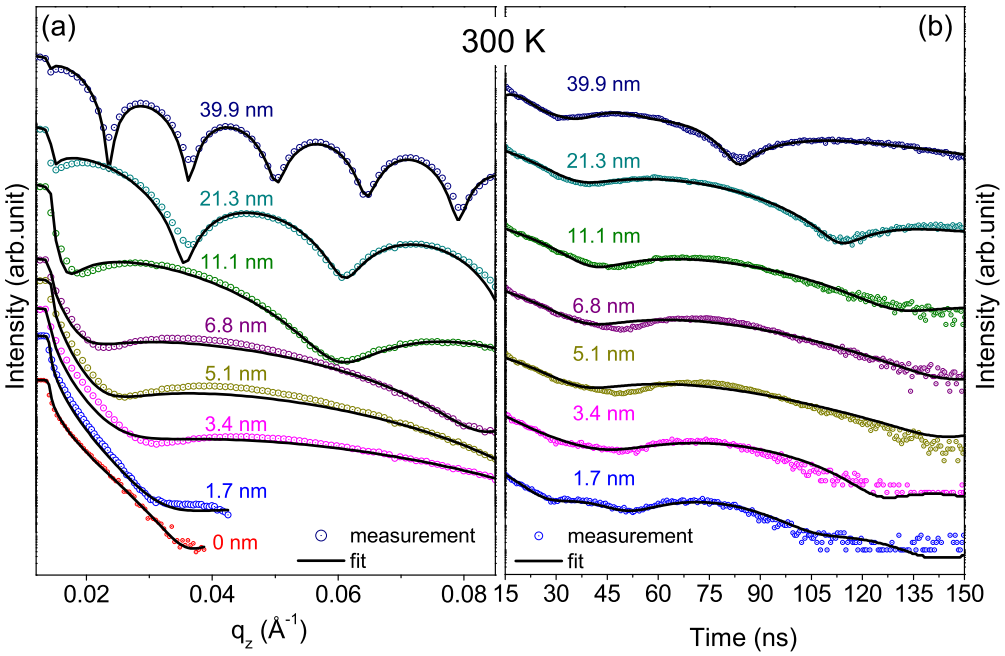

Fig. 3 X-ray reflectivity (a) and NRS pattern (b) of ${ }^{57} \mathrm{FeN}$ thin film grown in-situ on a sapphire substrate at room temperature $(300 \mathrm{~K})$. Measurements have been carried out using $14.4 \mathrm{keV}$ x-rays.

smaller than the longitudinal coherence length of the synchrotron beam [63,82]. However, since in our case two components (one S and one D) are present, the difference in their IS $(\triangle \mathrm{IS})$ can be used to compare fitting parameters obtained from the CEMS and the NRS measurements. For the thickest sample, we can find they show a similar behavior and grossly agree albeit few minor differences in $\triangle \mathrm{IS}, \mathrm{QS}$ and R.A. of S and D. As we can see that small differences in hyperfine parameters can be seen among samples grown under different conditions, therefore they can be understood from the fact that the CEMS measurement was carried out on an ex-situ grown sample whereas the NRS measurements were performed in-situ.

Using this model, the NRS spectra of thinner samples were also fitted as shown in fig. 3(b). Taking the thickest sample as a reference, we can see (from table 2) as film thickness reduces, the R.A. of $S$ increase while that if $D$ decrease, $\triangle I S$ and QS are increasing. For the thinnest sample $(1.7 \mathrm{~nm})$, the values of $\triangle \mathrm{IS}$ and QS are significantly large. It may be noted that when the thickness of the film is very small, typically below the percolation limit (about $2 \mathrm{~nm}$ for Fe), the film comprises of isolated grains and therefore the stress produced during the growth may lead to distortion in the cubic symmetry resulting in larger QS. Emergence of such QS was also observed in ultrathin Fe films during the in-situ growth of Fe films on $\mathrm{MgO}(001)$ [83] at $300 \mathrm{~K}$. The gradual reduction in QS with increase in the thickness indicates that the system transforms towards cubic symmetry which is also evident from our N $K$-edge XANES measurements presented in section 3.3. More details about the influence of film thickness on such stress are discussed in section 3.4. 
Table 2 Fitting parameters obtained from fitting of $300 \mathrm{~K}$ NRS data. Here, R.A. is the relative area of singlet (S) or doublet (D), QS denotes to quadrupole splitting, $\triangle \mathrm{IS}$ is the difference between IS of S and D.

\begin{tabular}{|c|c|c|c|c|}
\hline $\begin{array}{c}\text { Thickness } \\
\text { (nm) } \\
( \pm 0.5)\end{array}$ & $\begin{array}{c}\text { R.A. (S) } \\
(\%) \\
( \pm 1)\end{array}$ & $\begin{array}{c}\text { R.A. (D) } \\
(\%) \\
( \pm 1)\end{array}$ & $\begin{array}{c}\triangle \mathrm{IS} \\
(\mathrm{mm} / \mathrm{s}) \\
( \pm 0.05)\end{array}$ & $\begin{array}{c}\mathrm{QS} \\
(\mathrm{mm} / \mathrm{s}) \\
( \pm 0.05)\end{array}$ \\
\hline 1.7 & 43 & 57 & 1.54 & 1.50 \\
\hline 3.4 & 44 & 56 & 0.68 & 0.84 \\
\hline 5.1 & 52 & 48 & 0.48 & 0.79 \\
\hline 6.8 & 40 & 60 & 0.49 & 0.86 \\
\hline 11 & 32 & 68 & 0.55 & 0.76 \\
\hline 21.3 & 31 & 69 & 0.54 & 0.70 \\
\hline 39.9 & 30 & 70 & 0.47 & 0.68 \\
\hline
\end{tabular}
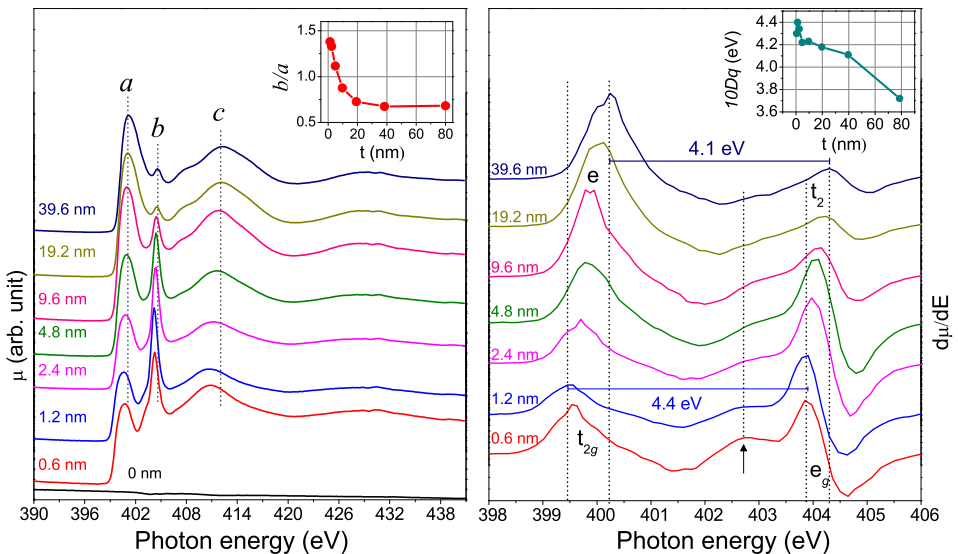

Fig. 4 N $K$-edge XANES measurements performed in-situ during the growth of FeN film on a Si substrate at $300 \mathrm{~K}$ using reactive magnetron sputtering.

\subsection{In-situ N $K$-edge XANES measurements}

Figure 4 shows the $\mathrm{N} K$-edge XANES patterns taken during the in-situ growth of FeN films at $300 \mathrm{~K}$. Before starting the deposition, the chamber was flushed with $\mathrm{N}_{2}$ gas 2-3 times and after evacuating the chamber to UHV conditions, $\mathrm{N} K$-edge measurements were performed on the bare substrate. As expected no signatures of N $K$-edge features can be seen on the bare substrate. Subsequently, FeN film was deposited for a short time and it was astonishing to see that prominent $\mathrm{N}$ $K$-edge features can be seen even when film thickness was less than a monolayer (not shown) and for a $0.6 \mathrm{~nm}$ film, well-resolved $\mathrm{N} K$-edge features can be seen in fig. 4(a). The assignment of these features as $a, b$ and $c$ was already explained in sec. 3.1. Shown in fig. 4(b) is a magnified view of the derivative of absorption coefficient $(\mathrm{d} \mu / \mathrm{dE})$ depicting the precise positions of features $a$ and $b$. For ultrathin films $(<5 \mathrm{~nm})$, we can see that the feature $b$ is the strongest, also it shows a shoulder on the lower energy side (marked by an arrow in fig. 4(b)), which could be due to some splitting in the $\mathrm{e}_{g}$ orbitals.

When the film thickness increases to $9.6 \mathrm{~nm}$, this shoulder disappears and the feature $b$ suddenly becomes the weakest. For films of even higher thickness the 
feature $b$ becomes even smaller (than both $a$ and $c$ ). The ratio of features $a$ and $b$ is plotted in the inset of fig. 4(a). Another prominent change that can be clearly seen is the shift in the position of features $a$ and $b$. We can see that both $a$ and $b$ shift to higher energy as thickness increases and the energy difference of these two features $(10 D q)$ reduces from $4.4 \mathrm{eV}$ to $4.1 \mathrm{eV}$ when the thickness increases from 1.2 to $39.6 \mathrm{~nm}$. This difference becomes even smaller $(\approx 3.7 \mathrm{eV})$ when the film thickness become even larger at about $80 \mathrm{~nm}$ (not shown). A variation in the energy separation $10 D q$ as a function of film thickness is shown in the inset of $4(\mathrm{~b})$.

The intensity ratio and the energy separation of the first two features of ligand $K$-edge (e.g. $\mathrm{O}$ or $\mathrm{N}$ ) in a transition metal oxide or nitride can be used to probe the hybridization between metal $3 d$ and $\mathrm{N}$ or $\mathrm{O} 2 p$ orbitals [62]. The energy separation $10 D q$ is a direct measure of ligand-field splitting and as atomic number increases $d$-orbitals become more extended resulting in larger overlap with $2 p$ orbitals and therefore $10 D q$ increases. It may be noted that $\mathrm{N} K$-edge XANES of TiN is well-studied and typically, $10 D q$ is about $2.5 \mathrm{eV}[68,76]$ and in thick FeN films it comes around $3.7 \mathrm{eV}$, this larger value of $10 D q$ in $\mathrm{FeN}$ (as compared to TiN) can be understood in terms extended $d$ orbitals in FeN. Even larger value of $10 D q \approx 4.4 \mathrm{eV}$ was observed in ultrathin FeN films. It can be understood if we assume that the symmetry of $\mathrm{N}$ atoms changes from tetrahedral (ZB-type) to octahedral (RS-type). Due to crystal field splitting five degenerate 3d orbitals split into $\mathrm{t}_{2 g}$ and $\mathrm{e}_{g}$ orbitals. In an octahedral symmetry, the three $\mathrm{t}_{2 g}$ orbitals points towards the corner and the two $\mathrm{e}_{g}$ orbitals towards the center of the cube. Therefore, the interaction of $\mathrm{e}_{g}$ orbitals with a ligand $(\mathrm{N})$ is stronger than those of $t_{2 g}$ orbitals. On the other hand, in a tetrahedral symmetry, the crystal field splitting is reversed, and $e$ orbitals are lower in energy than those of $t_{2}$ orbitals. Since in case of tetrahedral coordination, the symmetry is non-centrosymmetric, the gerade $(g)$ label is removed. Therefore, the reduction in $10 D q$ with an increase in film thickness can be understood if the structure changes from RS-type FeN in ultrathin films to ZB-type FeN in thicker films.

On the other hand, variations in the intensity ratios of first two features $b$ and $a$ are even more prominent across the films thickness, in particularly between film thickness of 1 to $10 \mathrm{~nm}$. The structural transition from RS-type to ZB-type could be one factor that influences the relative intensity of features $a$ and $b$, but the sharpness of the feature $b(\mathrm{FWHM} \approx 0.7 \mathrm{eV})$ and $b>>a$, is somewhat puzzling. Such an intense feature in N $K$-edge has been observed during the adsorption of nitrogen on $\mathrm{Mo}(110)$ surface and has been explained in terms of $\mathrm{N}-\mathrm{N}$ single and $\mathrm{N} \equiv \mathrm{N}$ triple bonds that are formed on the surface of Mo [62]. Polarizationdependent measurements of $\mathrm{N} K$-edge at the normal and grazing incidence by Chen et al. [62] evidenced a similar sharp feature resulting due to formation of $\mathrm{N}-\mathrm{N}$ single bond perpendicular to the surface. The presence of such sharp N $K$-edge feature can also be seen during oxidation of TiN [76] and also after the implantation of $\mathrm{N}^{+}$ions on $\mathrm{Zr}$ and $\mathrm{Hf}[76,62]$. In fact such $\mathrm{N}-\mathrm{N}$ or $\mathrm{N} \equiv \mathrm{N}$ bonds are also expected to be present as polymeric $\mathrm{N}$ chains in $\mathrm{N}$ rich $\mathrm{FeN}_{\mathrm{x}}(\mathrm{x}>1)$, evidenced recently under HPHT by Bykov et al. [16]. Recently Wu et al. [17] predicted the existence of $\mathrm{FeN}_{4}$ phase even under ambient pressure and temperature. The occurrence of such a sharp N $K$-edge feature may also stem from the presence of polymeric N chains in ultrathin FeN films but this needs to be further confirmed. The observed changes in the N $K$-edge XANES clearly demonstrate that ultrathin FeN films 


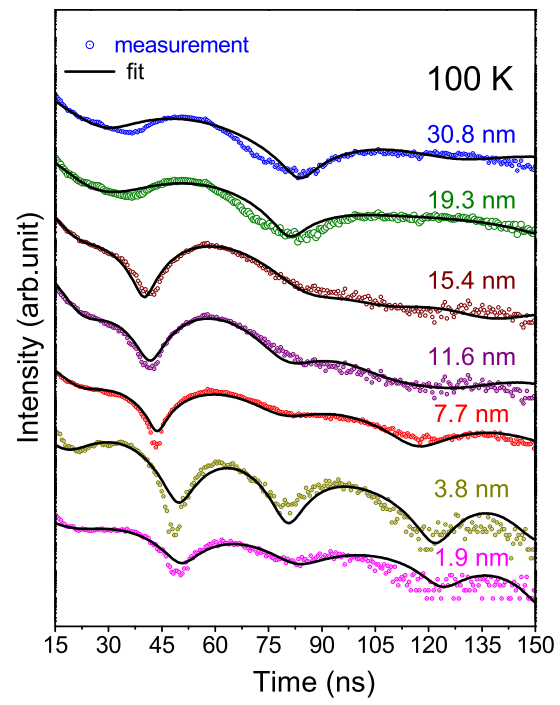

Fig. 5 NRS spectra of ${ }^{57} \mathrm{FeN}$ thin films grown in-situ at $100 \mathrm{~K}$ on a sapphire substrate.

$(<5 \mathrm{~nm})$ show a completely different behavior than rather thick FeN films and indicate a possibility about a structural transition from RS to ZB-type FeN around a thickness range of $5-10 \mathrm{~nm}$.

\subsection{In-situ NRS measurements at $100 \mathrm{~K}$}

From our room temperature $(300 \mathrm{~K})$ in-situ NRS, XRR, and N $K$-edge XANES measurements, the information obtained can be summarized as (i) irrespective of the thickness, FeN films were non-magnetic at $300 \mathrm{~K}$ (ii) the N $K$-edge XANES pattern of ultrathin films $(<5 \mathrm{~nm})$ show a different characteristics indicating that films may undergo a structural transition from RS to ZB-type as thickness increases beyond $5-10 \mathrm{~nm}$. As evidenced by Yamada et al. [42], the RS-type FeN has a AFM ground state with $\mathrm{T}_{\mathrm{N}} \approx 220 \mathrm{~K}$, therefore by doing NRS measurements at low temperatures (below $220 \mathrm{~K}$ ) it should be possible to verify if films become magnetic as expected for FeN in the RS-type structure.

Ideally, to compare such structural and magnetic transformations, it is required that films should be grown at the suitable temperature so as to attain the required long range ordering and then the NRS measurements should be done at low temperatures. This is normally possible using a close cycle refrigerator but due to limited space, in-situ growth and NRS measurements can not be normally done at low temperatures. A simplest way is to cool down the sample holder to the lowest possible temperature in a $\mathrm{LN}_{2}$ reservoir and then to study the in-situ growth directly at low temperatures. As expected, we found that film grown at $100 \mathrm{~K}$ was amorphous from XRD analysis (not shown). However, even then the local magnetic order is expected to remain similar as both an amorphous [84] and a crystalline [34] FeN shows a similar Mössbauer spectrum. 
Table 3 Fitting parameters obtained from fitting of $100 \mathrm{~K}$ NRS data for ${ }^{57} \mathrm{FeN}$ films of different thicknesses. Here, the component $\mathbf{C}_{1}$ has a finite values of hyperfine field $\left(\mathbf{B}_{\mathrm{hf}}\right)$ and QS and for the component $\mathbf{C}_{2}$, both $\mathbf{B}_{\mathrm{hf}}$ and QS $=0$ and $\triangle \mathrm{IS}$ is the difference between isomer shift of $\mathbf{C}_{1}$ and $\mathbf{C}_{2}$.

\begin{tabular}{cccccc}
\hline \hline $\begin{array}{c}\text { Thickness } \\
(\mathrm{nm})\end{array}$ & $\begin{array}{c}\text { R.A. }\left(\mathbf{C}_{1}\right) \\
(\%)\end{array}$ & $\begin{array}{c}\text { R.A. }\left(\mathbf{C}_{2}\right) \\
(\%)\end{array}$ & $\begin{array}{c}\mathbf{B}_{\mathrm{hf}}\left(\mathbf{C}_{1}\right) \\
(\mathrm{T})\end{array}$ & $\begin{array}{c}\mathrm{QS}\left(\mathbf{C}_{1}\right) \\
(\mathrm{mm} / \mathrm{s})\end{array}$ & $\begin{array}{c}\triangle \mathrm{IS} \\
(\mathrm{mm} / \mathrm{s})\end{array}$ \\
$( \pm 0.2)$ & $( \pm 1)$ & $( \pm 1)$ & $( \pm 0.5)$ & $( \pm 0.05)$ & $( \pm 0.05)$ \\
\hline 1.9 & 67 & 33 & 9.5 & 1.8 & 0.20 \\
3.8 & 61 & 39 & 9.7 & 1.8 & 0.18 \\
7.7 & 73 & 27 & 3.3 & 1.7 & 0.15 \\
11.6 & 74 & 26 & 3.1 & 1.3 & 0.16 \\
15.4 & 78 & 22 & 3.1 & 1.2 & 0.15 \\
19.3 & 79 & 21 & 3.3 & 0.7 & 0.11 \\
30.8 & 81 & 19 & 3.4 & 0.6 & 0.10 \\
\hline \hline
\end{tabular}

Similar to the procedures described for in-situ NRS measurements at $300 \mathrm{~K}$, the growth of FeN films was also studied by cooling the substrate holder by a continuous flow of $\mathrm{LN}_{2}$. Prior to cooling the sample holder, the whole system was baked to achieve UHV conditions which is an essential requirement to avoid condensation of moisture on the substrate surface at low temperatures. The estimated temperature at the sample surface was about $100 \mathrm{~K}$ and films of different thicknesses were deposited at this temperature. Here again total thickness was estimated from XRR pattern (not shown) and the deposition rate comes out to be $0.13 \mathrm{~nm} / \mathrm{s}$, about twice than that at $300 \mathrm{~K}$. Such enhancement in deposition rates is not unexpected at $\mathrm{LN}_{2}$ temperatures as evidenced recently during the growth of nickel oxide thin films [85].

The NRS spectra of FeN films grown at $100 \mathrm{~K}$ is shown in fig. 5 along with their best fit. They can be described with distinguished features as: (i) a broad oscillation in appearing in samples with thickness $\geq 7.7 \mathrm{~nm}$ (ii) narrower oscillations in ultrathin films $(\leq 7.7 \mathrm{~nm})$. Here, the broad oscillation corresponds to dynamical beats(DBs) $[86,87]$ originating due to the total thickness of sample and also due to IS and QS. With an increase in thickness DBs shift to lower time in agreement with the behavior that can also be seen in samples deposited at $300 \mathrm{~K}$ (fig. 3(b)). The additional beating pattern in ultrathin films $(\leq 7.7 \mathrm{~nm})$ can emerge due to presence of additional hyperfine interactions - electric quadrupole leading to QS or magnetic dipole resulting in Zeeman splitting. In both cases the nuclei deexcitation process results in interference amplitudes giving rise to oscillations in the scattered intensity with time. These oscillations are known as quantum beats (QBs) [88]. The period and the amplitude of QBs reveal the information about the magnetic structure present in the sample. These QBs are quite clearly visible in the 1.9 and $3.8 \mathrm{~nm}$ sample, and their presence, though weak, can also be seen in films of higher thicknesses.

The NRS spectra were fitted (using REFTIM software [81]) assuming two components (i) $\mathbf{C}_{1}$ - a component with a finite value of $\mathbf{B}_{\mathrm{hf}}$ and QS (ii) $\mathbf{C}_{2}$ - a non-magnetic component so that both $\mathbf{B}_{\mathrm{hf}}$ and $\mathrm{QS}=0$. Fitting parameters are given in table 3 . As can be seen from this table, on increasing the film thickness (i) R.A. of $\mathbf{C}_{1}$ increases while that of $\mathbf{C}_{2}$ descreases (ii) $\mathbf{B}_{\mathrm{hf}}$ and QS values of $\mathbf{C}_{1}$ decreases (iii) $\triangle \mathrm{IS}$ is quite small and decrease a bit. Obtained results clearly indicate the presence of a magnetic component with $\mathbf{B}_{\mathrm{hf}} \approx 10 \mathrm{~T}$ in ultrathin films 
(1.9 and $3.8 \mathrm{~nm}$ ) and $\mathbf{B}_{\mathrm{hf}}$ becomes very when thickness exceeds to $7.7 . \mathrm{nm}$ and does not change thereafter. The behavior of QS is almost similar to the growth at $300 \mathrm{~K}$ and can be understood along similar lines. It may be noted that our samples are polycrystalline (at $300 \mathrm{~K}$ ) or amorphous (at $100 \mathrm{~K}$ ), therefore the direction of $\mathbf{B}_{\mathrm{hf}}$ and electric field gradient is expect to be random and was considered likewise in the fitting. Further, it can se seen that overall values of $\triangle \mathrm{IS}$ are much smaller at $100 \mathrm{~K}$ as compared to those at $300 \mathrm{~K}$ and can be understood from the fact that such reduction in IS was also seen from in the low temperature CEMS spectra of thick FeN films [34].

The appearance of a magnetic component with $\mathbf{B}_{\mathrm{hf}}=9.5 \mathrm{~T}$ can be explained in terms of RS-type structure or some distortion in the ZB-type structure when film thickness is less below $7.7 \mathrm{~nm}$. It is expected that RS type FeN will have a AFM structure with $\mathbf{B}_{\mathrm{hf}} \approx 30 \mathrm{~T}[42,58]$. However, the value of $\mathbf{B}_{\mathrm{hf}}$ at $9.5 \mathrm{~T}$ is considerably smaller in our case. Since samples in this study have been deposited at $100 \mathrm{~K}$, it can be anticipated that crystalline ordering may not get established due to lack of adatom mobility and therefore in this situation structural disorders may lead to a smaller $\mathbf{B}_{\mathrm{hf}}$.

A close correlation can be drawn between the N $K$-edge XANES and the $100 \mathrm{~K}$ NRS spectrum of ultrathin $(\leq 7.7 \mathrm{~nm})$ FeN thin films. At such a low thickness, their $10 D q$ value is large $(4.4 \mathrm{eV})$, the ratio of features $b / a$ is also large and they are antiferromagnetic. The octahedral coordination between $\mathrm{Fe}$ and $\mathrm{N}$ atoms is indicative of the RS-type structure and the presence of AFM ground state further strengthen this observation. From the observed growth behavior, it appears that initially FeN films grow in the RS-type structure, subsequently due to an in plane tensile stress, films are forced to adopt the ZB-type structure. As already mentioned in section 1 , such structural evolutions were also evidenced by J. Pak et al. [47] from in-situ RHEED measurements in epitaxial FeN films grown using $\mathrm{N}$-plasma assisted MBE process. It was found that the LP was $4.52 \AA$ in the beginning and when the thickness exceeds about $20 \mathrm{~nm}$ it reduces to $4.32 \AA$. It seems that the critical thickness at which such transition takes place will depend upon the growth temperature, in our case films are grown at $300 \mathrm{~K}$ and such structural transition is taking at much lower thickness. It can be anticipated that by growing FeN films at high substrate temperature and then by studying the magnetic properties of ultrathin films at low temperatures, more precise estimation of magnetic ground state of FeN can be made.

\section{Conclusions}

In this work, the structural and the magnetic properties of ultrathin FeN films were studied during in-situ growth using the N K-edge XANES at $300 \mathrm{~K}$ and NRS measurements at 300 and $100 \mathrm{~K}$. The N $K$-edge XANES measurements clearly reveal that the coordination between Fe and $\mathrm{N}$ atoms changes from octahedral (RS-type) to tetrahedral (ZB-type) when thickness increases beyond $5 \mathrm{~nm}$. Low temperature NRS measurements also depict such behavior as ultrathin FeN films show a magnetic character. When the thickness of films increases beyond $5 \mathrm{~nm}$, films become non-magnetic as expected in for ZB-type FeN. The remarkable sensitivity of both XANES and NRS for ultrathin layers can be further utilized by growing FeN 
films at higher temperatures and then by studying the structural and magnetic properties at low temperatures.

Acknowledgements We would like to thank to the Department of Science and Technology, India for the financial support and Jawaharlal Nehru Centre for Advanced Scientific Research (JNCASR) for managing the project. We acknowledge technical help received from L. Behera, A. Gome and R. Sah. We are thankful to V. Ganesan and A. K. Sinha for support and encouragements.

\section{References}

1. S. Bhattacharyya, J. Phys. Chem. C 119, 1601 (2015).

2. M. Takahashi, H. Shoji, J. Magn. Magn. Mater. 208, 145 (2000).

3. Y. Jiang, M.A. Mehedi, E. Fu, Y. Wang, L.F. Allard, J.P. Wang, Sci. Rep. 6, 25436 (2016).

4. S. Kokado, N. Fujima, K. Harigaya, H. Shimizu, A. Sakuma, Phys. Rev. B 73, 172410 (2006).

5. A. Houari, S.F. Matar, M.A. Belkhir, J. Magn. Magn. Mater. 322, 658 (2010).

6. K. Ito, G.H. Lee, K. Harada, M. Suzuno, T. Suemasu, Y. Takeda, Y. Saitoh, M. Ye, A. Kimura, H. Akinaga, Appl. Phys. Lett. 98, 102507 (2011).

7. E.L.P.y. Blancá, J. Desimoni, N.E. Christensen, H. Emmerich, S. Cottenier, Phys. Status Solidi B 246, 909 (2009).

8. H. Li, X. Li, D. Kim, G. Zhao, D. Zhang, Z. Diao, T. Chen, J.P. Wang, Appl. Phys. Lett. 112, 162407 (2018).

9. W. Yin, D. Zhang, P. Zhang, X. Wang, W. Wang, X. Lei, Z. Shi, H. Yang, J. Alloys Compds. 688, 828 (2016).

10. H. Huang, S. Gao, A.M. Wu, K. Cheng, X.N. Li, X.X. Gao, J.J. Zhao, X.L. Dong, G.Z. Cao, Nano Energy 31, 74 (2017).

11. Y. Cai, A. Li, J. Cao, X. Ni, G. Zhang, G. Yu, W. Xu, Nucl. Instrum. Methods Phys. Res., Sect. B 168, 422 (2000).

12. D. Laniel, A. Dewaele, G. Garbarino, Inorg. Chem. 57, 6245 (2018).

13. Y.N. Xu, P. Rulis, W.Y. Ching, J. Appl. Phys. 91, 7352 (2002).

14. W.Y. Ching, Y.N. Xu, P. Rulis, Appl. Phys. Lett. 80, 2904 (2002).

15. M. Wessel, R. Dronskowski, Chem. Eur. J. 17, 2598 (2011).

16. M. Bykov, E. Bykova, G. Aprilis, K. Glazyrin, E. Koemets, I. Chuvashova, I. Kupenko, C. McCammon, M. Mezouar, V. Prakapenka, H.P. Liermann, F. Tasnádi, A.V. Ponomareva, I.A. Abrikosov, N. Dubrovinskaia, L. Dubrovinsky, Nat. Commun. 9, 2756 (2018).

17. L. Wu, R. Tian, B. Wan, H. Liu, N. Gong, P. Chen, T. Shen, Y. Yao, H. Gou, F. Gao, Chem. Mater. 30, 8476 (2018).

18. K.H. Jack, Proceedings of the Royal Society of London. Series A. Mathematical and Physical Sciences 208, 200 (1951).

19. A. Oueldennaoua, E. Bauer-Grosse, M. Foos, C. Frantz, Scripta Metall. 19, 1503 (1985).

20. H. Nakagawa, S. Nasu, H. Fujii, M. Takahashi, F. Kanamaru, Hyperfine Interact. 69, 455 (1991).

21. K. Suzuki, H. Morita, T. Kaneko, H. Yoshida, H. Fujimori, J. Alloys Compds. 201, 11 (1993)

22. T. Hinomura, S. Nasu, Physica B 237-238, 557 (1997).

23. P. Schaaf, C. Illgner, M. Niederdrenk, K.P. Lieb, Hyperfine Interact. 95, 199 (1995)

24. M. Niederdrenk, P. Schaaf, K.P. Lieb, O. Schulte, J. Alloys Compds. 237, 81 (1996).

25. L. Rissanen, M. Neubauer, K.P. Lieb, P. Schaaf, J. Alloys Compds. 274, 74 (1998).

26. P. Schaaf, Prog. Mater. Sci. 47, 1 (2002).

27. M. Gupta, A. Gupta, S. Chaudhari, D.M. Phase, V. Ganesan, M.R. Rao, T. Shripathi, B. Dasannacharya, Vacuum 60, 395 (2001).

28. X. Wang, W. Zheng, H. Tian, S. Yu, W. Xu, S. Meng, X. He, J. Han, C. Sun, B. Tay, Appl. Sur. Sci. 220, 30 (2003).

29. D. Peng, T. Hihara, K. Sumiyama, J. Alloys Compds. 377, 207 (2004).

30. R. Gupta, M. Gupta, Phys. Rev. B 72, 024202 (2005).

31. V. Demange, T.H. Loi, P. Weisbecker, E. Bauer-Grosse, Thin Solid Films 494, 184 (2006). 
32. I. Jouanny, P. Weisbecker, V. Demange, M. Grafouté, O. Peña, E. Bauer-Grosse, Thin Solid Films 518, 1883 (2010).

33. M. Gupta, A. Tayal, A. Gupta, R. Gupta, J. Stahn, M. Horisberger, A. Wildes, J. Appl. Phys. 110, 123518 (2011).

34. M. Gupta, A. Tayal, A. Gupta, V. Reddy, M. Horisberger, J. Stahn, J. Alloys Compds. $\mathbf{5 0 9}, 8283$ (2011).

35. A. Tayal, M. Gupta, A. Gupta, P. Rajput, J. Stahn, Phys. Rev. B 92, 054109 (2015).

36. A. Tayal, M. Gupta, N. Pandey, A. Gupta, M. Horisberger, J. Stahn, J. Alloys Compds. 650, 647 (2015).

37. K. Jantasom, M. Horprathum, P. Eiamchai, S. Limwichean, N. Nuntawong, V. Patthanasettakul, P. Chindaudom, C. Thanachayanont, P. Songsirirtthigul, Mater. Today:. Proc. 4, 6173 (2017).

38. Q. Lu, M. Xie, G. Han, B. Zheng, Y. Song, J. Qiang, X. Wang, Z. Wu, P. Yan, W. Liu, J. Magn. Magn. Mater. 474, 76 (2019).

39. M. Gupta, A. Gupta, P. Bhattacharya, P. Misra, L. Kukreja, J. Alloys Compds. 326, 265 (2001).

40. F. Sun, J. Liu, Y. Yang, H. Yu, Mater. Sci. Eng., B 122, 29 (2005).

41. R. Usui, Y. Yamada, Y. Kobayashi, Hyperfine Interact. 205, 13 (2012).

42. Y. Yamada, R. Usui, Y. Kobayashi, Hyperfine Interact. 219, 13 (2013).

43. A. Tayal, M. Gupta, A. Gupta, V. Ganesan, L. Behera, S. Singh, S. Basu, Surf. Coat. Technol. 1, 2 (2015).

44. C. Navío, J. Alvarez, M.J. Capitan, F. Yndurain, R. Miranda, Phys. Rev. B 78, 155417 (2008).

45. D.M. Borsa, D.O. Boerma, Hyperfine Interact. 151, 31 (2003).

46. E. Andrzejewska, R. Gonzalez-Arrabal, D. Borsa, D. Boerma, Nucl. Instrum. Methods Phys. Res., Sect. B 249, 838 (2006).

47. J. Pak, W. Lin, K. Wang, A. Chinchore, M. Shi, D.C. Ingram, A.R. Smith, K. Sun, J.M. Lucy, A.J. Hauser, F.Y. Yang, J. Vac. Sci. Technol., A 28, 536 (2010).

48. M. Naito, K. Uehara, R. Takeda, Y. Taniyasu, H. Yamamoto, J. Cryst. Growth 415, 36 (2015).

49. K. Niwa, T. Terabe, D. Kato, S. Takayama, M. Kato, K. Soda, M. Hasegawa, Inorg. Chem. 56, 6410 (2017).

50. D. Laniel, A. Dewaele, S. Anzellini, N. Guignot, J. Alloys Compds. 733, 53 (2018).

51. W.P. Clark, S. Steinberg, R. Dronskowski, C. McCammon, I. Kupenko, M. Bykov, L. Dubrovinsky, L.G. Akselrud, U. Schwarz, R. Niewa, Angewandte Chemie International Edition 56, 7302 (2017).

52. M. Zheng, X. Chen, R. Cheng, N. Li, J. Sun, X. Wang, T. Zhang, Catal. Commun. 7, 187 (2006).

53. Z. Yang, S. Guo, X. Pan, J. Wang, X. Bao, Energy Environ. Sci. 4, 4500 (2011).

54. C. Navío, J. Alvarez, M.J. Capitan, J. Camarero, R. Miranda, Appl. Phys. Lett. 94, 263112 (2009).

55. C. Navío, M.J. Capitán, J. Álvarez, R. Miranda, F. Yndurain, New J. Phys. 12, 073004 (2010)

56. S. Li, X. Cui, Z.F. Liu, T.L. Song, J. Alloys Compds. 771, 322 (2019).

57. Y. Cheballah, A. Ziane, S. Bouarab, A. Vega, J. Phys. Chem. Solids 100, 148 (2017).

58. Z. Zhao, K. Bao, D. Duan, F. Tian, B. Liu, T. Cui, RSC Adv. 5, 31270 (2015).

59. V.F. Hlynsson, E. Skúlason, A.L. Garden, J. Alloys Compds. 603, 172 (2014).

60. H.R. Soni, V. Mankad, S.K. Gupta, P.K. Jha, J. Alloys Compds. 522, 106 (2012).

61. A. Houari, S.F. Matar, M.A. Belkhir, M. Nakhl, Phys. Rev. B 75, 064420 (2007).

62. J.G. Chen, Surf. Sci. Rep. 30, 1 (1997).

63. R. Röhlsberger, Nuclear Condensed Matter Physics with Synchrotron Radiation, vol. 208 (Springer-Verlag, 2004).

64. D.M. Phase, M. Gupta, S. Potdar, L. Behera, R. Sah, A. Gupta, AIP Conference Proceedings 1591, 685 (2014).

65. Dynamics beamline p01 web site. http://photon-science.desy.de/facilities/petra_ iii/beamlines/p01_dynamics/index_eng.html

66. F. Aksoy, G. Akg J. Alloys Compds. 508, 233 (2010).

67. M. Tallarida, C. Das, D. Schmeisser, Beilstein J. Nanotechnol. 5, 77 (2014).

68. R. Gupta, M. Gupta, D.M. Phase, AIP Conference Proceedings 1832, 080081 (2017).

69. P. Vishwakarma, M. Gupta, D.M. Phase, A. Gupts, J. Phys.: Condens. Matter 31, 105001 (2019) 
70. H. Wille, H. Franz, R. Röhlsberger, W. Caliebe, F. Dill, in J. Phys. Conf. Ser., 217, 012008, (2010).

71. K. Schlage, R. Röhlsberger, J. Electron. Spectrosc. Relat. Phenom. 189, 187 (2013).

72. G.V. Smirnov, Hyperfine Interact. 97, 551 (1996).

73. R. Rüffer, Comptes Rendus Physique 9, 595 (2008).

74. R. Rüffer, A.I. Chumakov, Hyperfine Interact. 97, 589 (1996).

75. M. Naito, K. Uehara, R. Takeda, Y. Taniyasu, H. Yamamoto, J. Cryst. Growth 415, 36 (2015).

76. L. Soriano, M. Abbate, H. Pen, M. Czyzyk, J. Fuggle, J. Electron. Spectrosc. Relat. Phenom. 62, 197 (1993).

77. C. Mitterbauer, C. Hébert, G. Kothleitner, F. Hofer, P. Schattschneider, H. Zandbergen, Solid State Commun. 130, 209 (2004).

78. Y. Tripathi, R. Gupta, Seema, M. Gupta, D. Phase, P. Rajput, Thin Solid Films 670, 113 (2019).

79. E. Fratczak, J. Prieto, M. Moneta, Journal of Alloys and Compounds 586, 375 (2014)

80. C. Braun, Parratt32- The Reflectivity Tool (HMI Berlin, 1997-99).

81. M.A. Andreeva, Hyperfine Interact. 185, 17 (2008).

82. R. Lübbers, G. Wortmann, H.F. Grünsteudel, Hyperfine Interactions 123(1-4), 529 (1999)s

83. G. Sharma, A. Gupta, M. Gupta, K. Schlage, H.C. Wille, Phys. Rev. B 92, 224403 (2015).

84. M. Gupta, A. Gupta, S. Rajagopalan, A.K. Tyagi, Phys. Rev. B 65, 214204 (2002).

85. Y. Abe, S. Yamauchi, M. Kawamura, K.H. Kim, T. Kiba, J. Vac. Sci. Technol., A 36, $02 \mathrm{C} 102$ (2018).

86. U. van Bürck, W. Potzel, P. Schindelmann, Y.V. Shvyd́ko, E. Gerdau, O. Leupold, H. Rüter, Phys. Rev. A 61, 013803 (1999).

87. Y.V. Shvyd́ko, U. Van Bürck, W. Potzel, P. Schindelmann, E. Gerdau, O. Leupold, J. Metge, H. Rüter, G. Smirnov, Phys. Rev. B 57, 3552 (1998).

88. E. Gerdau, R. Rüffer, R. Hollatz, J. Hannon, Phys. Rev. Lett. 57, 1141 (1986). 